\title{
A Synthesis of Hempelian and Hypothetico-Deductive Confirmation
}

\author{
April 5, 2011
}

\begin{abstract}
This paper synthesizes confirmation by instances and confirmation by successful predictions, and thereby the Hempelian and the hypotheticodeductive traditions in confirmation theory. Our merger of these two approaches solves classical problems such as the tacking paradoxes and the raven paradox and is subsequently extended to the piecemeal confirmation of entire theories. We compare our proposal to rivalling suggestions and discuss its merits and drawbacks, especially vis-à-vis Bayesianism.
\end{abstract}




\section{Introduction}

This article presents a synthesis of hypothetico-deductive (H-D) and Hempelian confirmation and extends it to the piecemeal confirmation of entire theories. Such a project may sound anachronistic in a time where purely qualitative, first-order logic approaches in philosophy of science are considered a failure, and apparently superseded by more powerful quantitative accounts such as Bayesianism. Therefore, before actually embarking on the project itself, we consider it necessary to devote some lines to why we believe that this is a fruitful endeavor at all. (Readers who take this for granted are invited to skip a couple of paragraphs.)

There is a popular prejudice that with the advent and success of Bayesianism, qualitative accounts of confirmation have become obsolete. Bayesians model the beliefs of scientists by means of probability functions, and explicate degree of confirmation as the credibility boost that a tested hypothesis experiences in the face of the evidence. This seems to be a general, encompassing model that contains qualitative accounts as special cases. ${ }^{1}$

While we do not want to question the benefits of Bayesian reasoning for modeling inductive inference, we doubt that it is a complete theory. For instance, frequentist (rather than Bayesian) techniques still dominate uncertain reasoning in science, and this state of the art has, by some philosophers, been defended on methodological grounds (e.g., Mayo 1996). What is more, even those scientists that subscribe, foundationally, to Bayesianism sometimes refrain from subjective Bayesian inference because they do not consider it suitable for allegedly objective scientific communication (e.g., Bernardo 2011).

The visible restraint of scientists and methodologists to fully endorse Bayesianism fits the observation that increase in degree of belief is, in many cases in the history of science, a poor explanation of why a piece of evidence confirms a theory (Glymour 1980a). Think of Kepler's laws and Tycho Brahe's observations of the orbit of Mars, Lavoisier's refutation of the phlogiston theory in his experiments on combustion, or the General Theory of Relativity and Eddington's observations of the 1919 eclipse. In these and similar cases, Bayesianism is, rather than an explication of scientific confirmation, an instrument to measure its extent: it neither explains in virtue of what we raise our degree of belief in the tested theory, nor why scientists often show unanimous agreement on the interpretation of an experiment.

\footnotetext{
${ }^{1}$ See Kuipers (2000) for an extended discussion of qualitative vs. Bayesian confirmation theory.
} 
Clearly, Bayesianism is a very general and useful model of confirmation, and we have no ambition to play the anti-Bayesian here. But the above arguments, elaborated in much greater detail in the cited works, suggest that confirmation might, like explanation, be a plural notion. Thus, even a die-hard Bayesian need not dismiss the study of qualitative confirmation as superfluous. Quite to the contrary, by studying qualitative accounts, we can get an idea of what scientists refer to when talking about structural, objective relations between theory and evidence, and unravel why Bayesian reasoning is still struggling to find its place in many areas of science.

There are two grand traditions in qualitative confirmation theory. One prominent proposal for the structure of scientific confirmation has been made by William Whewell:

Our hypotheses ought to foretel phenomena which have not yet been observed $[\ldots]$ the truth and accuracy of these predictions were a proof that the hypothesis was valuable and, at least to a great extent, true. (Whewell 1847, 62-63)

Modeling empirical support by successful (deductive) prediction is the bottom line of the hypothetico-deductive model of confirmation. From the hypothesis under test and some auxiliary assumptions, we derive empirical predictions that confirm, if verified, the original hypothesis. For instance, a physicist will test the harmonic oscillator model captured by the equation $\ddot{x}+\omega^{2} x=0$ for swinging pendula by deriving its consequences for a particular pendulum. If the predictions of the oscillator model are verified, they confirm the harmonic oscillator model, if not, they refute it.

Moreover, the H-D model resembles the "conjectures and refutations"-model of scientific progress (Popper 1934/71): hypothesis have to be subjected to severe tests in order to gain corroboration. This fact distinguishes it among all qualitative accounts of confirmation. It is thereby an attractive model for those who are reluctant to assign degrees of belief in the truth of any particular hypothesis (a presumption of Bayesianism) but who believe that by subjecting a hypothesis to severe tests and failing to observe refutations, it can be corroborated and appraised over others.

The other grand tradition is confirmation by instances, or, for the sake of convenience, Hempelian confirmation. This goes back to Jean Nicod (1925) who modeled l'induction par confirmation as the discovery of instances of a hypothesis under test. See also Glymour (1980a). Planet orbits are instances of Kepler's laws. Swinging pendula instantiate the harmonic oscillator. Black 
ravens instantiate the hypothesis that all ravens are black. Hempel (1945/65) provided the first rigorous formalization of this idea by demanding that the evidence entail the development of the hypothesis to the domain of the evidence. This is quite different from the H-D account where the deductive arrow goes from the hypothesis to the evidence. Apparently, both approaches pursue very different accounts of confirmation, suggesting that it might be hard to come up with a unified account of qualitative confirmation.

Nevertheless, this is the project we undertake. The next section presents a couple of pertinent objections that impair either account. After arguing that these problems can be overcome by integrating a Hempelian and a H-D perspective on scientific confirmation, we generalize our account to the confirmation of networks of hypotheses, that is, scientific theories. We do not pretend to have solved all problems of qualitative confirmation theory and to have evaded all objections that have plagued previous accounts, but judging our proposal from its conceptual soundness, simplicity, and viability in paradigmatic cases, we think that a good case has been made. We conclude by arguing that it improves on previous suggestions and introduces a novel and interesting twist to theorizing about scientific confirmation.

\section{Classical Problems for Qualitative Accounts}

Classical formulations of H-D confirmation such as

(H-D) Evidence $E H$-D confirms hypothesis $H$ relative to background knowledge $K$ if and only if (1) H.K is consistent, (2) H.K entails $E$ and (3) $K$ alone does not entail $E$.

have several severe shortcomings. First, we often want to say that the results of a scientific experiment do not only support an isolated hypothesis, but speak in favor of an entire theory consisting of several interrelated models or theories (Dietrich and Moretti 2005). (H-D) does not specify how entire theories, or major parts thereof, are confirmed, as opposed to the confirmation of single hypotheses. Second, (H-D) is unable to cope with the so-called tacking paradoxes. It is possible to tack irrelevant conjunctions to the hypothesis $H$ and to preserve the confirmation relation: If $H$ is confirmed by a piece of evidence $E$ relative to $K, H . X$ is confirmed by the same $E$ relative to $K$, as long as $X$ is consistent with H.K. To use our initial example: If the predictions of the harmonic oscillator model about a swinging pendulum are verified, they would also confirm the harmonic oscillator model of the pendulum together with the 
hypothesis that all ravens are black. That is unacceptable. Vice versa, it is possible to tack irrelevant disjunctions to the evidence $E$ and to preserve the confirmation relation: If $E$ confirms a hypothesis $H$ relative to $K, E \vee E^{\prime}$ confirms the same $H$ for an arbitrary $E^{\prime}$, relative to $K$. So, equally unacceptably, the pendulum data or the observation of a single black raven would confirm that swinging pendula are harmonic oscillators. Both objections exploit the fact that any prediction, however partial it is, still counts as confirming, or in other words: classical H-D confirmation gives no account of evidential relevance. These failures of hypothetico-deductive confirmation might lead to the conclusion that the entire approach is hopeless and should be abandoned (Glymour 1980b).

Hempelian confirmation (Hempel 1945/65), on the other hand, is usually identified with the satisfaction criterion:

(Hempel) Evidence $E$ (directly) Hempel-confirms hypothesis $H$ relative to background knowledge $K$ if and only if $E . K$ entails the development of $H$ for $E$, that is, the restriction of $H$ to the set of singular terms that occur essentially in $E .^{2}$ Formally, the criterion amounts to $E . K \models H_{\mid E}$.

However, (Hempel) is exposed to equally strong, perhaps devastating criticism. (Hempel) is monotonous with respect to background knowlege, that is, the addition of more background knowledge cannot destroy the confirmation relation. This can lead to disastrous consequences. Consider the hypothesis $H=\forall x:(R x \rightarrow B x)$ that all ravens are black, and the evidence $E=\neg B a . \neg R a$ that we observe a non-black non-raven. Hempel (1945/65) makes a convincing case that such a piece of evidence may confirm the raven hypothesis as long as we do not know beforehand that $a$ is no raven: such observations rule out potential counterexamples to the raven hypothesis. For instance, if we observe a grey bird that resembles a raven, then finding out that it was a crow confirms the raven hypothesis.

However, Hempel's own account of confirmation is inconsistent with this analysis (Fitelson and Hawthorne 2010): relative to the background knowledge $K=\neg R a$ (" $a$ is no raven"), $E . K=\neg B a . \neg R a$ implies $H_{\mid E}=(R a \rightarrow B a)$. Although the color of birds known to be crows or swans cannot tell us anything about the truth of the raven hypothesis, $E$ Hempel-confirms $H$ relative to $K$ in this example, creating a clearly unacceptable confirming instance.

\footnotetext{
${ }^{2}$ Definition 2 will make this notion precise; see Hempel (1943) for the original definition.
} 
Finally, it is worrying that Hempelian confirmation is so silent about the wellentrenched idea that hypotheses are confirmed by successful predictions. Given the significance of that approach both in scientific practice and in philosophical theorizing, it is awkward that (Hempel) remains silent on that point. The remainder of the paper is devoted to tackling the problems outlined in this section.

\section{Irrelevant Disjunctions: Content Parts}

The source of the problem of irrelevant disjunction is the property of first-order logic that well-formed forms (wffs) sometimes have irrelevant consequences: for instance, the conclusion in $F a \models(F a \vee G a)$ contains the irrelevant element $G a$. We need a logical relation stronger than deductive entailment to discern irrelevant disjuncts. For the sake of simplicity, we presuppose a first order predicate language $L$ without identity, but the extensions are straightforward. ${ }^{3}$

Ken Gemes' (1993) account of content parts achieves that goal by analyzing relevance relations between wffs. The following definition captures our intuitive view of relevance relations between two wffs:

Definition 1 An atomic well-formed form (wff) $\beta$ is relevant to a wff $\alpha$ if and only if there is some model $M$ of $\alpha$ such that: if $M^{\prime}$ differs from $M$ only in the value $\beta$ is assigned, $M^{\prime}$ is not a model of $\alpha$.

Intuitively, $\beta$ is relevant for $\alpha$ if at least in one model of $\alpha$ the truth value of $\beta$ cannot be changed without making $\alpha$ false. In other words, the truth value of $\alpha$ is not fully independent of the truth value of $\beta$. A particularly interesting application of this account of relevance is the notion of the domain and the development of a wff.

Definition 2 The domain of a well-formed formula $\alpha$ is the set of singular terms that occur in the atomic wffs that are relevant for $\alpha$. The development of a universally quantified wff $\alpha$ for another wff $\beta$, written $\alpha_{\mid \beta}$, is the restriction of $\alpha$ to the domain of $\beta$, that is, we evaluate the truth value of $\alpha$ with respect to the domain of $\beta$.

For instance, the domain of $F a . F b$ is $\{a, b\}$ whereas the domain of $F a . G a$ is $\{a\}$, and the development of $\forall x: F x$ for $F a . \neg G b$ is $F a . F b$.

Moreover, we can define the notion of a relevant model which assigns truth values to all and only the relevant atomic wffs:

\footnotetext{
${ }^{3}$ A generalization of the content part relation to richer languages that can be used for $\mathrm{H}-\mathrm{D}$ confirmation, e.g. languages with identity, is given in Gemes (1997).
} 
Definition $3 A$ relevant model of a wff $\alpha$ is a model of $\alpha$ that assigns truth values to all and only those atomic wffs that are relevant to $\alpha$.

So relevant models remain silent on the truth values of irrelevant atomic wffs. This allows us to define the notion of a content part, where in addition to logical entailment, all relevant models of the consequens can be extended to relevant models of the antecedens:

Definition 4 For two wffs $\alpha$ and $\beta, \beta$ is a content part of $\alpha\left(\alpha \models_{c p} \beta\right)$ if and only if (1) $\alpha$ and $\beta$ are contingent, (2) $\alpha$ logically entails $\beta$ and (3) every relevant model of $\beta$ has an extension which is a relevant model of $\alpha$.

The content part relation forbids irrelevant disjunctions in the conclusion. For instance, $F a \vee G a$ is no content part of $F a$ because the model that assigns 'false' to $F a$ and 'true' to $G a$ is a relevant model of $F a \vee G a$, but no model of $\mathrm{Fa}$. The content part relation marks such deductions as irrelevant. Following Gemes (1993), we can improve on our original definition of H-D confirmation by postulating

(H-D*) Evidence $E H$-D confirms hypothesis $H$ relative to background knowledge $K$ if and only if (1) $H . K$ is consistent, (2) $E$ is a content part of $H . K\left(H . K \models_{c p} E\right)$ and (3) $K$ alone does not entail $E$.

\section{Irrelevant Conjunctions: Synthesizing Hempel and H-D}

Unfortunately, (H-D*) does not solve all tacking paradoxes: the problem of irrelevant conjunctions persists. The pendulum data still confirm the hypothesis that pendula are harmonic oscillators and that all ravens are black. To rule out these problems, Gemes (1993) has taken refuge in a quite complicated account of natural axiomatizations of a theory.

That strategy has its merits, but also its drawbacks (Schurz 2005). First, it is not always clear which axiomatizations should count as "natural" and which not. Second, Gemes' account is rather complicated and hard to interpret intuitively. Keeping in mind Carnap's $(1950, \S 3)$ requirement that explications should be as simple as possible, we might decide to look for alternatives. Unfortunately, the available suggestions (e.g., Schurz 1991) are not without problems either (Gemes 1998).

We propose to combine content parts and modus tollens in order to discern irrelevant conjunctions. The basic idea of H-D confirmation - that $E$ is a prediction of $H$ - can be expressed in two ways: $H \models E$ and $\neg E \models \neg H$. The latter 
formulation states that failure to observe a prediction refutes a hypothesis, or in other words, the evidence has to put the hypothesis to a serious test. If we qualify this entailment by means of content parts and restrict it to the domain of the evidence - that is, the set of singular terms that are relevant to $E$-, then the problem of irrelevant conjunctions is solved by demanding that

$$
\neg E . K \models_{c p} \neg H_{\mid E} . K .
$$

If $H$ is the compound of a 'relevant' and an 'irrelevant' hypothesis, then the content part relation will not hold between $\neg E$.K and $\neg H_{\mid E}$. $K$, because the irrelevant conjunctions have been transformed into irrelevant disjunctions. For example, if $H=\forall x:(F x \cdot G x), E=F a$, and $K=\top$, then $\neg H_{\mid E} \cdot K=\neg F a \vee \neg G a$ is no content part of $\neg E . K=\neg F a$.

(1) can also be interpreted as a Hempelian requirement on successful confirmation. The problem of irrelevant conjunctions occurs when evidence produces only partial instances of a hypothesis: $F a$ is no instance of $H=\forall x:(F x \cdot G x)$, etc. Since Hempel's satisfaction criterion required that $E$ be a full instance of $H$, it did not suffer from that problem. Our move in (1) accounts for that intuition and exploits that if $E$ is no instance of $H$, such as in the above irrelevant conjunction case, then $\neg H_{\mid E}$. $K$ will typically be no content part of $\neg E$.K.

Hence, we can suggest a definition of qualitative confirmation that synthesizes Hempelian and H-D confirmation:

(Syn) Evidence $E$ confirms hypothesis $H$ relative to background knowledge $K$ if and only if

- $E$ is a content part of $H . K\left(H . K \models_{c p} E\right)$, and

- $\neg H_{\mid E} . K$ is a content part of $\neg E . K\left(\neg E . K \models_{c p} \neg H_{\mid E} . K\right)$.

(Syn) successfully copes with the tacking paradoxes, and in doing so, it improves upon classical H-D confirmation as well as upon Hempel's proposal. For instance, in the raven paradox, both $E_{1}=B a$ and $E_{2}=\neg R a$ confirm $H=\forall x:(R x \rightarrow B x)$, relative to $K_{1}=R a$ and $K_{2}=\neg B a$, respectively. This is in line with Hempel's resolution of the paradox, namely to bite the bullet. But notably, $H$ is no more confirmable by known non-ravens whose color is subsequently observed, as it used to be the case in Hempel's own account. Nicod, Hempel and Glymour were right about the significance of instances for the confirmation of a hypothesis - they did not see, though, the prospects of marrying that view with hypothetico-deductive confirmation. 
(Syn) does not yet explain how different parts of a theory can be confirmed by a body of composite evidence. This feature of (Syn) is particularly salient if we examine the behavior of that account with respect to the confirmation of several hypotheses at once. Assume that a biologist conducts a couple of experiments with a cell culture. Unfortunately, she can use each cell only once, that is, for one experiment. Reasonably, she partitions the cell culture into different groups and performs experiment A with group 1, experiment B with group 2, and so on. If the experiments are successful, they should, taken together, confirm the conjunction of the hypotheses. In other words, if $E_{1}$ confirms $H_{1}$ and $E_{2}$ confirms $H_{2}$, then $E_{1} . E_{2}$ should also confirm $H_{1} . H_{2}$.

Unfortunately, our present account violates that desideratum. For instance, $E=F a . G b$ will not confirm the hypothesis $H=\forall x:(F x . G x)$ relative to $K=\mathrm{T}$. Thus, while (Syn) synthesizes Hempelian and H-D confirmation, we are lacking an extension where the confirmation of independent hypotheses can add up to the confirmation of an entire theory which is composed of the former.

\section{An Extension to Theory Confirmation}

For extending (Syn) to the confirmation of entire theories, we let ourselves once more inspire by Hempel's approach. For Hempel, a theory is confirmed if it is entailed by a set of sentences that are individually confirmed by the evidence. Following this idea, we propose to construct a 1:1-match of theory and evidence: theories are decomposed into their content parts which are, individually, confirmed by a specific content part of the evidence. If all content parts of the theory are confirmed in this way, the entire theory is confirmed. For example, assume that we would like to confirm Kepler's Three Laws by means of observing the planetary orbits in the solar system. Then we use the position of a single planet at different points in time to confirm the first two laws, whereas we require data about the orbital period and the semi-major axis of at least two different planets in order to confirm the Third Law.

Furthermore, by building on the (Syn) criterion from the previous section, we avoid the tacking paradoxes. In other words, we stipulate that evidence $E$ confirms a theory $T$ if (i) we can derive that evidence from the theory (relative to background knowledge), and (ii) there is a decomposition of $T$ into content parts $H_{1}, \ldots, H_{n}$ such that for each $H_{i}$, the evidence contains an instance of $H_{i}$.

We can condense this reasoning into the following definition:

Definition 5 Evidence $E$ confirms theory $T$ relative to background knowledge 
$K$ if and only if

- $E$ is a content part of $T . K\left(T . K \models{ }_{c p} E\right)$

- There are wffs $H_{1}, \ldots, H_{n}$ such that $H_{1}, \ldots, H_{n} \models T$ and for all $i \leq n$, $T \models{ }_{c p} H_{i}$, and there is a wff $E_{i}$ such that

$-E \models{ }_{c p} E_{i}$

$-\neg E_{i} . K \models_{c p} \neg\left(H_{i}\right)_{\mid E_{i}} . K$, that is, $\neg\left(H_{i}\right)_{\mid E_{i}} . K$ is a content part of $\neg E_{i} . K$.

To illustrate how definition 5 works, consider a medical trial. We would like to test the theory $T$ that only plasmodium parasites cause malaria in humans. More precisely, the theory consists of the individual hypotheses $H_{1}, H_{2}, H_{3}$, etc. that only plasmodium parasites can cause the different forms of malaria $M_{1}, M_{2}$, $M_{3}$. We test these hypothesis by scrutinizing patients that have been suffering of malaria, sorting them into subtrials according to the kind of malaria $M_{i}$. If the individual trials confirm the hypothesis $\left(T . K \models_{c p} E, \neg E_{i} . K \models_{c p} \neg\left(H_{i}\right)_{\mid E_{i}} . K\right)$, then we have also confirmed our overarching theory, since the evidence of each trial $E_{i}$ is a content part of the total evidence. Furthermore, definition 5 solves our biologist's problem: if two different properties ( $F$ and $G$ ) are supposed to be demonstrated of a population, we can decompose the composite hypothesis $\forall x: F x . G x$ into its content parts $\forall x: F x$ and $\forall x: G x$, each of which is confirmed by a content part of the evidence $(F a$ and $G b)$.

Summing up, Definition 5 has a number of desirable implications. It solves the tacking paradoxes, gives an account of how entire theories can be confirmed in a piecemeal fashion, and does so using only a single technical concept: content part entailment, a refinement of deductive entailment. A fortiori, we can also apply it to the confirmation of single hypotheses.

\section{Discussion and Conclusion}

In this paper, we have synthesized Hempelian and H-D confirmation, that is, confirmation by instances and confirmation by successful predictions. We have contended that the reputation of qualitative confirmation as either hopeless or outdated is unjustified: it captures important structural relations between theory and evidence, and it can be immunized against the classical objections. The main competitor on the quantitative side - Bayesianism - is an attractive framework for modeling learning under uncertainty, but it does not fully explain why degrees of belief are changed in a certain way, why scientists agree so 
often on the confirmatory status of a specific experiment. Therefore, qualitative confirmatory arguments have a place in scientific reasoning.

By integrating Hempelian confirmation into the hypothetico-deductive account, we have solved the tacking paradoxes, extended our account to the confirmation of entire theories, and retained a convincing solution to the ravens' paradox. However, marrying the two has consequences that one may find unattractive, too. Confirming existential statements, and universal statements without finite models remains difficult. ${ }^{4}$

Another objection would contend that our account is limited to theories with purely observational content: since the evidence must be stated in terms of observational properties, it is hard to see how $\neg H_{\mid E}$ (that may refer to unobservable properties) can ever be a content part of $\neg E$. For instance, suppose that an electron is shot into an electromagnetic field. It will then experience a Lorentz force and change its direction accordingly. Then, $\neg H_{\mid E}$ seems to be a disjunction of an observable and a non-observable proposition. So it cannot be a content part of the (purely observational) $\neg E$.

This objection is only valid if there are no logical inference relations between unobservable and observable properties. But if the electron experiences an electromagnetic force, it will be deflected orthogonally to its original direction and to the electromagnetic force lines. This follows directly from the formula for the Lorentz force $\vec{F}=q(\vec{v} \times \vec{B})$. Conversely, if the electron fails to move in that direction, we can infer that there cannot have been an (unobservable) Lorentz force, and we can infer from $\neg E$ to $\neg H_{\mid E}$. Thus, the proposal also applies to theories with partly unobservable content.

On a whole, our proposal is simpler and more straightforward than the rivalling suggestions of Gemes (1993) and Schurz (1991), and it gives a satisfactory treatment of paradigmatic problems such as the tacking paradoxes, the raven paradox, and the confirmation of entire theories. We don't say that it is entirely unproblematic, we don't say that contrived counterexamples cannot be constructed. Still, we conclude that our account makes a considerable net surplus: we are now able to reconcile two conflicting intuitions and to explain why successful predictions and instances of an hypothesis both have confirmatory force, while at the same time solving the classical paradoxes and modeling the piecemeal confirmation of entire theories.

\footnotetext{
${ }^{4}$ Moreover, a hypothesis such as $H=\forall x, y: R x y$ is not confirmed by $R a b$ relative to tautologous background knowledge because $R a b$ does not constitute a full instance of $H$. For that, we would require to observe $R a a, R b a$, and $R b b$ as well. Thus, one may ask whether the intuitive pull of confirmation by instances applies to monadic predicates only - a question that is left to future work.
} 


\section{References}

Bernardo, José M. (2011): "Integrated Objective Bayesian Estimation and Hypothesis Testing" (with discussion), in J. M. Bernardo et al. (eds.): Bayesian Statistics 9: Proceedings of the Ninth Valencia International Meeting, 1-68. Oxford: Oxford University Press.

Carnap, Rudolf (1950): Logical Foundations of Probability. Chicago: The University of Chicago Press.

Dietrich, Franz, and Luca Moretti (2005): "On Coherent Sets and the Transmission of Confirmation", Philosophy of Science 72, 403-424.

Fitelson, Branden, and James Hawthorne (2010): "How Bayesian Confirmation Theory Handles the Paradox of the Ravens", in: Ellery Eells and James Fetzer (ed.), The Place of Probability in Science, 247-276. New York: Springer.

Gemes, Ken (1993): "Hypothetico-Deductivism, Content and the Natural Axiomatisation of Theories", Philosophy of Science 60, 477-487.

Gemes, Ken (1997): "A New Theory of Content II: Model Theory and Some Alternatives", Journal of Philosophical Logic 26, 449-476.

Gemes, Ken (1998): "Hypothetico-Deductivism: The Current State of Play", Erkenntnis 49, 1-20.

Gemes, Ken (2006): “Content and Watkins' Account of Natural Axiomatizations", dialectica 60 , 85-92.

Glymour, Clark (1980a): Theory and Evidence. Princeton: Princeton University Press.

Glymour, Clark (1980b): "Discussion: Hypothetico-Deductivism is Hopeless", Philosophy of Science 47, 322-325.

Hempel, Carl G. (1943): "A Purely Syntactical Definition of Confirmation", Journal of Symbolic Logic 8, 122-143.

Hempel, Carl G. (1945/65): "Studies in the Logic of Confirmation", in: Aspects of Scientific Explanation, 3-46. The Free Press, New York. Reprint from Mind 54, 1945.

Kuipers, Theo (2000): From Instrumentalism to Constructive Realism. Dordrecht: Kluwer. 
Mayo, Deborah G. (1996): Error and the Growth of Experimental Knowledge. Chicago and London: The University of Chicago Press.

Nicod, Jean (1925): Le problème logique de l'induction. Paris: Alcan.

Popper, Karl R. (1934/71): Logik der Forschung. Third Edition. Tübingen: Mohr.

Schurz, Gerhard (1991): "Relevant Deduction", Erkenntnis 35, 391-437.

Schurz, Gerhard (2005): "Bayesian H-D Confirmation and Structuralistic Truthlikeness: Discussion and Comparison with the Relevant-Element and the Content-Part Approach", in: Roberto Festa (ed.), Logics of Scientific Discovery. Essays in Debate with Theo Kuipers, 141-159. Amsterdam: Rodopi.

Whewell, William (1847): Philosophy of the Inductive Sciences, Founded Upon Their History. Vol. II. London: Parker. 